\title{
Frank Decker
}

\section{Kosmopolitismus versus Kommunitarismus: eine neue Konfliktlinie in den Parteiensystemen?}

Zusammenfassung: Der starke Zulauf rechtspopulistischer Parteien wird von manchen Autoren auf die Herausbildung eines vermeintlich neuen Cleavages in den Parteiensystemen zurückgeführt, das zwischen kosmopolitischen und kommunitaristischen Positionen verlaufe. Bei Lichte betrachtet handelt es sich dabei jedoch nur um eine Reformulierung der vorhandenen ökonomischen und kulturellen Konfliktlinien, die begrifflich unscharf bleibt und keinen theoretischen Mehrwert bietet. Um die Veränderung der Parteiensysteme zu verstehen, sollte der Blick auf das jeweilige Gewicht der ökonomischen und kulturellen Konflikte sowie auf deren Wechselbeziehungen gerichtet werden.

Schlïsselwörter: Parteiensysteme, Cleavages, Kosmopolitismus, Kommunitarismus, Rechtspopulismus

Frank Decker, Cosmopolitanism versus communitarianism: a new line of conflict in party systems?

Summary: The strong electoral appeal of right-wing populism is attributed by some authors to a supposed new cleavage in party systems which distinguishes between cosmopolitan and communitarian attitudes. At closer inspection, however, the new model is only a reformulation of the existing economic and cultural cleavages which remains conceptually vague and adds nothing to the established theories. If we want to understand party system change, it is necessary to look at the respective relevance of the economic and cultural conflicts and their mutual relations.

Keywords: Party systems, cleavages, cosmopolitism, communitarianism, right wing populism

Frank Decker, Dr. phil., Dipl. pol., Professor am Institut für Politische Wissenschaft und Soziologie der Universität Bonn und wissenschaftlicher Leiter der Bonner Akademie für Forschung und Lehre praktischer Politik (BAPP)

Korrespondenzanschrift: frank.decker@uni-bonn.de 
Als Seymour Martin Lipset und Stein Rokkan ${ }^{1}$ vor mehr als fünfzig Jahren ihre berühmt gewordene These von den »eingefrorenen « Konfliktlinien prägten, konnten sie nicht vorausahnen, wie schnell die anschließende Entwicklung der Parteiensysteme in den westlichen Demokratien ihre Theorie auf die Probe stellen würde. Deren in den siebziger Jahren einsetzender und sich in den kommenden Jahrzehnten weiter beschleunigende Wandel war äußerlich einerseits an der nachlassenden Wählerunterstützung der Parteien des rechten und linken Mainstreams ablesbar. Andererseits betraten neue - ökologische, rechtspopulistische und regionalistische - Parteien die Bühne, von denen sich viele dauerhaft etablierten.

Stand der wieder aufkommende Regionalismus in direktem Gegensatz zu Lipsets und Rokkans modernisierungstheoretisch geprägten Vermutung, wonach territoriale Gegensätze in den Industriegesellschaften zugunsten »funktionaler « Konflikte an Bedeutung verlieren würden, so wurden die ökologischen und rechtspopulistischen Parteien in der Literatur vielfach als Folge neuer Konfliktlinien gedeutet, die die bestehenden, laut Lipset / Rokkan für die Herausbildung der Parteiensysteme im 19. und 20. Jahrhundert beiden hauptverantwortlichen Cleavages - das religiös-konfessionelle und das Klassencleavage - ergänzt hätten und quer zu diesen verliefen.

Ähnlich wie die historischen Cleavages, deren Genealogie Lipset und Rokkan in ihrem Aufsatz auf faszinierende Weise dargestellt hatten, bildeten sich die neuen Cleavages in einer - allerdings kürzeren - zeitlichen Abfolge. Fiel die Entstehungszeit der meisten grünen Parteien in die siebziger und frühen achtziger Jahre, so verteilten sich die rechtspopulistischen Neugründungen über einen langen Zeitraum, nämlich von Mitte der achtziger bis in die 2010er Jahre - mit der »Alternative für Deutschland « als prominentestem Spätankömmling. Die dänischen und norwegischen Vertreter waren als Vorreiter sogar bereits in den siebziger Jahren entstanden. ${ }^{2}$

Blickt man auf die Wahlergebnisse, lässt sich ein deutlicher Aufschwung der Rechtspopulisten in den 2010er Jahren feststellen. Dieser korrespondiert mit zusammengenommen noch höheren Verlusten der christlich-konservativen und sozialdemokratischen Parteien, wobei die letzteren davon relativ stärker betroffen sind, weil sie die Stimmen nicht nur im eigenen Lager - an Grüne und Linksparteien - abgeben, sondern in erheblichen Maße auch an die Rechtspopulisten. Es dürfte nicht zuletzt dieses erklärungsbedürftige Phänomen sein, dass einen Teil der Parteien- und Demokratieforscher inspiriert hat, den Aufstieg des Rechtspopulismus einer vermeintlich neuen, erst im letzten Jahrzehnt virulent gewordenen Konfliktlinie zuzuschreiben, die auf die Folgen der beschleunigten Globalisierung zurückgehe und begrifflich an den Gegenpolen Kosmopolitismus und Kommunitarismus festgemacht werden könne.

1 Seymour Martin Lipset / Stein Rokkan (Hg.), Party Systems and Voter Alignments. Cross-National Perspectives, New York 1967.

2 Vgl. Frank Decker, Parteien unter Druck. Der neue Rechtspopulismus in den westlichen Demokratien, Opladen 2000. 
Laut Wolfgang Merkel ${ }^{3}$ lässt sich dieser neue Konflikt auf die Frage herunterbrechen, ob (und wieweit) die Grenzen der Nationalstaaten geöffnet oder geschlossen werden (bleiben) sollen. Die »Grenzfrage« beziehe sich dabei »umfassend auf Güter, Dienstleistungen, Kapital, Arbeitskräfte, Flüchtlinge, Menschenrechte oder aber die Abgabe nationalstaatlicher Kompetenzen zugunsten supranationaler Regime und transnationaler Politik. « ${ }^{4}$ Sie habe also eine ökonomische und kulturelle Dimension. Während die Kosmopoliten wirtschafts- und gesellschaftspolitisch liberale Positionen miteinander verbänden, vereinten die Kommunitaristen das ökonomische Schutzbedürfnis des Sozial- und Wohlfahrtsstaates mit dem politischen Selbstbestimmungsbedürfnis einer kulturell definierten Gemeinschaft. Die wichtigste Trennlinie bilde dabei die Sicht auf die Nation. Von einem universalistischen Begriff der Menschenrechte ausgehend, wollten die Kosmopoliten diese nach innen für fremde und globale Einflüsse öffnen und nach außen durch eine zwischenstaatliche, im Idealfalle supranationale $\mathrm{Zu}$ sammenarbeit überwinden. Der Globalisierung stünden sie prinzipiell positiv gegenüber. Die Kommunitaristen hielten dagegen an einem partikularistischen Verständnis der Kultur und politischen Zugehörigkeit fest. Sie betrachteten die Globalisierung skeptisch und erwarteten von der nationalstaatlichen Politik, dass sie die Bürger vor deren vermeintlich negativen Auswirkungen schütze.

So plausibel diese Gegenüberstellung auf den ersten Blick anmutet, sind Zweifel angebracht, ob sie wirklich eine neue Konfliktlinie begründet, die einen zusätzlichen, das heißt über die vorhandenen Cleavagetheorien hinausreichenden Erklärungsbeitrag für die jüngsten Veränderungen der Parteiensysteme leistet.

Der erste Einwand stützt sich auf die soziologische Untermauerung des Kosmopolitismus-Kommunitarismus-Gegensatzes. Merkel verweist selbst darauf, dass die wirtschaftliche Interessenlage hier wenig erkläre. Es seien vor allem Humankapital, also Bildung, und kulturelles Kapital, die der Herausbildung kosmopolitischer Einstellungen zugrunde lägen. Insofern handelt es sich im Kern um eine kulturelle Konfliktlinie, deren Bedeutungsanstieg den Übergang von der Industriegesellschaft zur heutigen Wissensgesellschaft widerspiegelt. Wurde die Trennlinie zwischen Arbeiterklasse und Bürgertum in der Industriegesellschaft hauptsächlich vom ökonomischen Kapital bestimmt, hängt die soziale Schichtung in der Wissensgesellschaft vor allem vom kulturellen Kapital ab, das an die Seite (nicht unbedingt an die Stelle) des Geldeinkommens tritt. Wie sich dieser Strukturwandel der Moderne in der Lebens- und Arbeitswelt niederschlägt, hat der Soziologie Andreas Reckwitz ${ }^{5}$ in seinem Buch »Gesellschaft der Singularitäten « prägnant beschrieben.

3 Ähnlich gelagert ist die Unterscheidung der »Somewheres « und »Anywheres « des britischen Publizisten David Goodhart. Vgl. ders., The Road to Somewhere. The Populist Revolt and the Future of Politics, London 2017, S. $19 \mathrm{ff} .$.

4 Wolfgang Merkel, »Kosmopolitismus versus Kommunitarismus. Ein neuer Konflikt in der Demokratie« in: Philipp Harfst / Ina Kubbe Thomas Poguntke (Hg.), Parties, Governments and Elites, Wiesbaden 2017 S. 9.

5 Andreas Reckwitz, Die Gesellschaft der Singularitäten. Zum Strukturwandel der Moderne, Berlin 2017. 
Der zweite Einwand bezieht sich auf die Feststellung, dass sich ökonomische und kulturelle Trennlinien im Kosmopolitismus-Kommunitarismus-Gegensatz überlappten. Auch das ist nichts Neues, sondern in der Cleavagetheorie ein »alter Hut«. Das Phänomen sich überkreuzender Konfliktlinien wurde in der Ära der klassischen Cleavages vor allem im Verhältnis von religiös-konfessioneller und ökonomischer Klassenzugehörigkeit sichtbar. Katholische Arbeiter, wählten, wenn sie kirchengebunden waren, in der Frühzeit der Bundesrepublik eher CDU als SPD. Das änderte sich erst, als im Zuge der Säkularisierung das religiöse gegenüber dem ökonomischen Cleavage an Bedeutung verlor. Auch für die heutige Hinwendung vieler sozialdemokratischer oder linker Wähler zum Rechtspopulismus hatte die von Lipset $^{6}$ geprägte Formel des working class authoritarianism in den fünfziger Jahren bereits eine treffende Erklärung bereit gehalten.

Letzteres verweist auf einen weiteren wichtigen Punkt. Zu den zentralen Feststellungen Lipsets und Rokkans gehörte, dass gesellschaftliche Konflikte nicht von sich aus zur Parteien- und Lagerbildung führten, sondern erst, wenn sie durch vorhandene Eliten politisch mobilisiert würden. Dasselbe gilt für ihren weiteren Bestand. Sind die Eliten eine Verbindung mit einem bestimmten Teil der Wählerschaft eingegangen, müssen sie diese Verbindung stets aufs Neue aktualisieren und pflegen. ${ }^{7}$ Auch hier bietet die von Merkel in Übereinstimmung mit anderen Stimmen in der Rechtspopulismusforschung diagnostizierte »Repräsentationslücke ${ }^{8}$ keinen theoretischen Mehrwert. Die darin enthaltene Behauptung, dass sich die etablierten Parteien in ihrem programmatischen und personellen Angebot von den Präferenzen ihrer potenziellen Nachfrager entfernt hätten, ist einerseits trivial. Andererseits möchte sie suggerieren, dass diese Entfernung zugleich eine Folge der eigenen lebensweltlichen Abgehobenheit der Eliten sei, die - aus der aufgestiegenen Mittelschicht stammend - ein kosmopolitisches Weltbild teilten, selbst wenn Teile von ihnen aus wahlstrategischen Gründen auch kommunitaristische Positionen verträten. ${ }^{9}$

Dies führt zu einem vierten und letzten Argument, das an der Begrifflichkeit der vermeintlich neuen Konfliktlinie ansetzt. Auch als Idealtypen gedacht, stellen diese eine groteske Überzeichnung, um nicht zu sagen Karikatur eines Gegensatzes dar, der in Wirklichkeit eine Schimäre ist, weil ja die Menschen immer beides anstreben: individuelle Selbstentfaltung und gemeinschaftliche Einbindung. So wie sich viele »Kosmopoliten « zugleich nach sozialer Erdung sehnen ${ }^{10}$ und durchaus restriktiv auftreten können, wenn die Öffnung für das Andere ihr eigenes Umfeld direkt betrifft, stehen

6 Vgl. Seymour Martin Lipset, Political Man. The Social Bases of Politics, New York 1960, S. $97 \mathrm{ff}$.

7 Vgl. Karl Rohe, Wablen und Wäblertraditionen in Deutschland. Kulturelle Grundlagen deutscher Parteien und Parteiensysteme im 19. und 20. Jabrhundert, Frankfurt a.M. 1992, S. 25.

8 Vgl. z.B. Werner J. Patzelt, »Der 18. Deutsche Bundestag und die Repräsentationslücke. Eine kritische Bilanz« in: Zeitschrift für Staats- und Europawissenschaften 16, Nr.2/3 (2017), S. 245-285.

9 Merkel, Kosmopolitismus versus Kommunitarismus, aaO. (FN 4), S. 16.

10 Wie der Elitenforscher Michael Hartmann ermittelt hat, gilt dies selbst für die Spitzengruppe der kosmopolitischen Jetset-Eliten. So seien von den aus Deutschland stammenden CEOs 
die meisten »Kommunitaristen « kultureller Vielfalt und Zuwanderung positiv gegenüber, solange sie das Gefühl haben, die Kontrolle über die Öffnungsprozesse und damit über ihre eigene Lebenswelt zu behalten. ${ }^{11}$ Die Begriffe liegen insofern auf unterschiedlichen Ebenen. Die eigentliche Trennlinie verläuft nicht zwischen Kosmopoliten und Kommunitaristen, sondern innerhalb der beiden Gruppen - zwischen wirtschaftsliberalen Verfechtern des globalen Wettbewerbs und Vertretern eines wertebezogenen, globalen Humanismus auf der einen Seite sowie zwischen einem identitären und liberalen Kommunitarismus auf der anderen Seite.

Fasst man die Einwände zusammen, weist der Gegensatz von Kosmoplitismus und Kommunitarismus über das in der Parteienforschung seit den neunziger Jahren weithin akzeptierte »neue Konfliktlinienmodell nicht wirklich hinaus, das - an die funktionale Dimension in Lipsets und Rokkans Schema anschließend - zwischen einem sozioökonomischen (verteilungsbezogenen) und einem kulturellen (wertebezogenen) Cleavage unterscheidet. Vom klassischen Modell hebt sich dieses vereinfachte Schema erstens darin ab, dass es bei der Betrachtung der Konfliktlinien weniger auf die Beziehungen der Parteien zu bestimmten gesellschaftlichen Gruppen rekurriert als auf die von ihnen vertretenen ideologisch-programmatischen Grundpositionen. Nachdem sich die Interessenlagen und Wertvorstellungen der Bürger in der nachindustriellen Gesellschaft von den harten Merkmalen der Sozialstruktur immer mehr abgelöst haben, werden diese Positionen auf der Wählerseite heute in erster Linie durch Einstellungsmerkmale reflektiert. Und zweitens tritt an die Stelle des religiös-konfessionellen jetzt ein allgemeines kulturelles Cleavage, bei dem libertäre Haltungen wie Toleranz, nonkonformistisches Denken und Multikulturalität autoritären Haltungen wie Ordnungsdenken, Festhalten an konventionellen Lebensformen und Nationalstolz gegenüberstehen.

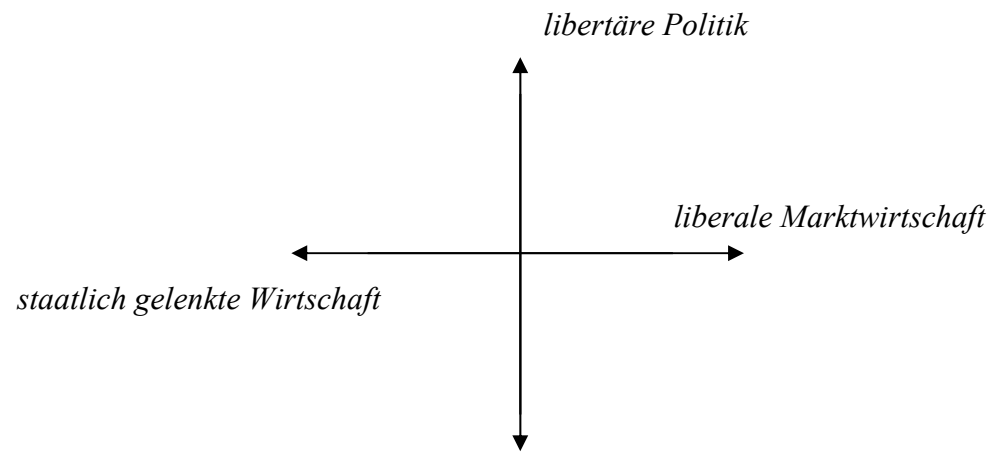

autoritäre Politik

ausländischer Unternehmen der größte Teil nicht in den USA oder Asien anzutreffen, sondern in Österreich und der Schweiz - also innerhalb des eigenen Sprach- und Kulturraums. Vgl. Michael Hartmann, Die globale Wirtschaftselite. Eine Legende, Frankfurt a.M. 2016.

11 Vgl. Thomas Meyer, "Heimat, Nation - Sozialdemokratie?« in: Die Neue Gesellschaft / Frankfurter Hefte 65, Nr. 11 (2018), S. 40. 
Wenn man an diesem Schema ${ }^{12}$ Kritik üben wollte, müsste sie auch hier zuerst an der Begrifflichkeit ansetzen. Autoritäre Positionen markieren nur eine Extremform der zuletzt genannten Haltungen, die man deshalb allgemeiner und weniger pejorativ als »konservativ« bezeichnen sollte. Noch problematischer erscheint die Verwendung des Begriffs »libertär « für die Gegenposition, denn mit diesem wird zugleich eine radikale Spielart des Marktliberalismus assoziiert, die systematisch eher der ökonomischen Konfliktlinie zuzuordnen ist. Sinnvoller wäre es, allgemeiner von liberalen Positionen zu sprechen.

Ein zweiter Einwand knüpft daran unmittelbar an. So wie das Schema in den politikwissenschaftlichen Lehrbüchern heute nahezu unisono abgebildet wird, erweckt es den Eindruck, dass die Links-Rechts-Unterscheidung sich ausschließlich auf das ökonomische Cleavage beziehe und das kulturelle Cleavage von ihr nicht berührt werde beziehungsweise quer dazu liege. Dies ist nicht nur deshalb fragwürdig, weil das neue Konfliktlinienschema ja gerade auf die ideologischen Positionen der politischen Anbieter Bezug nehmen will. Es widerspricht auch der politikwissenschaftlichen Intuition, die für die kulturell liberalen Positionen den Begriff »linksliberal« ebenso selbstverständlich verwendet wie sie die konservativen oder autoritären Positionen als »Rechtspopulismus « apostrophiert.

Maßgeblich für die Unterscheidung zwischen Links und Rechts ist das Gleichheitsverständnis. »Das Thema, das in allen Variationen wiederkehrt, ist das des Gegensatzes zwischen horizontaler oder egalitärer Vision der Gesellschaft und vertikaler oder nicht-egalitärer Vision. «13 Dieser Gegensatz lässt sich sowohl auf der ökonomischen als auch auf der kulturellen Konfliktachse abbilden. Ökonomisch geht es um die materielle Gleichheit. Die Rechten betrachten die Ergebnisse des Marktes im Prinzip als gerecht, während die Linken sie mithilfe staatlicher Umverteilung zugunsten der Schwächeren korrigieren möchten. Kulturell geht es um rechtliche Gleichheit. Setzt sich die Linke hier für einen umfassenden Abbau von Benachteiligungen gesellschaftlicher Gruppen und Minderheiten ein, halten die Rechten bestimmte Ungleichbehandlungen mit Verweis auf natürliche Unterschiede (des Geschlechts-, der ethnisch-kulturellen Zugehörigkeit oder der sexuellen Orientierung) durchaus für vertretbar.

Um das Konfliktlinienmodell mit der Links-Rechts-Unterscheidung zu verbinden, gibt es grafisch zwei Möglichkeiten. Entweder trägt man den wirtschaftlichen und kulturellen Links-Rechts-Gegensatz horizontal auf derselben Achse ab, dann ergibt sich das folgende Schema mit vier Quadranten. ${ }^{14}$

12 Das Schaubild findet sich bei Uwe Jun, »Repräsentation durch Parteien. Die Entwicklung des deutschen Parteiensystems und Parteienwettbewerbs nach 1945 « in: Carsten Koschmieder (Hg.), Parteien, Parteiensysteme und politische Orientierungen, Wiesbaden 2017, S. 92.

13 Noberto Bobbio, Rechts und Links. Gründe und Bedeutung einer politischen Unterscheidung, Berlin 1994, S. 74.

14 Decker, Parteien unter Druck, aaO. (FN 2), S. 304, modifizierte Darstellung. 


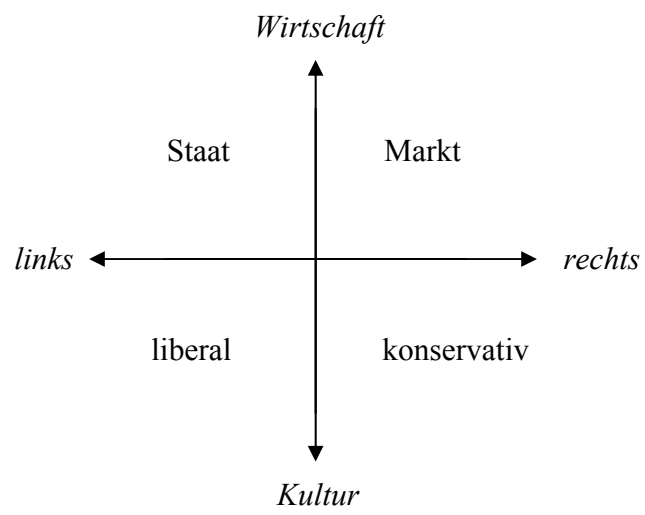

Oder man kippt das Schema mit der horizontal aufgetragenen ökonomischen und vertikal aufgetragenen kulturellen Konfliktachse im Uhrzeigersinn in die Diagonale. Die frei bleibenden Quadranten können dann genutzt werden, um zu markieren, welche Positionen die Parteien in einem bestimmten Parteiensystem auf der ökonomischen und kulturellen Achse jeweils einnehmen. ${ }^{15}$ Dass die Links-Rechts-Zuordnung

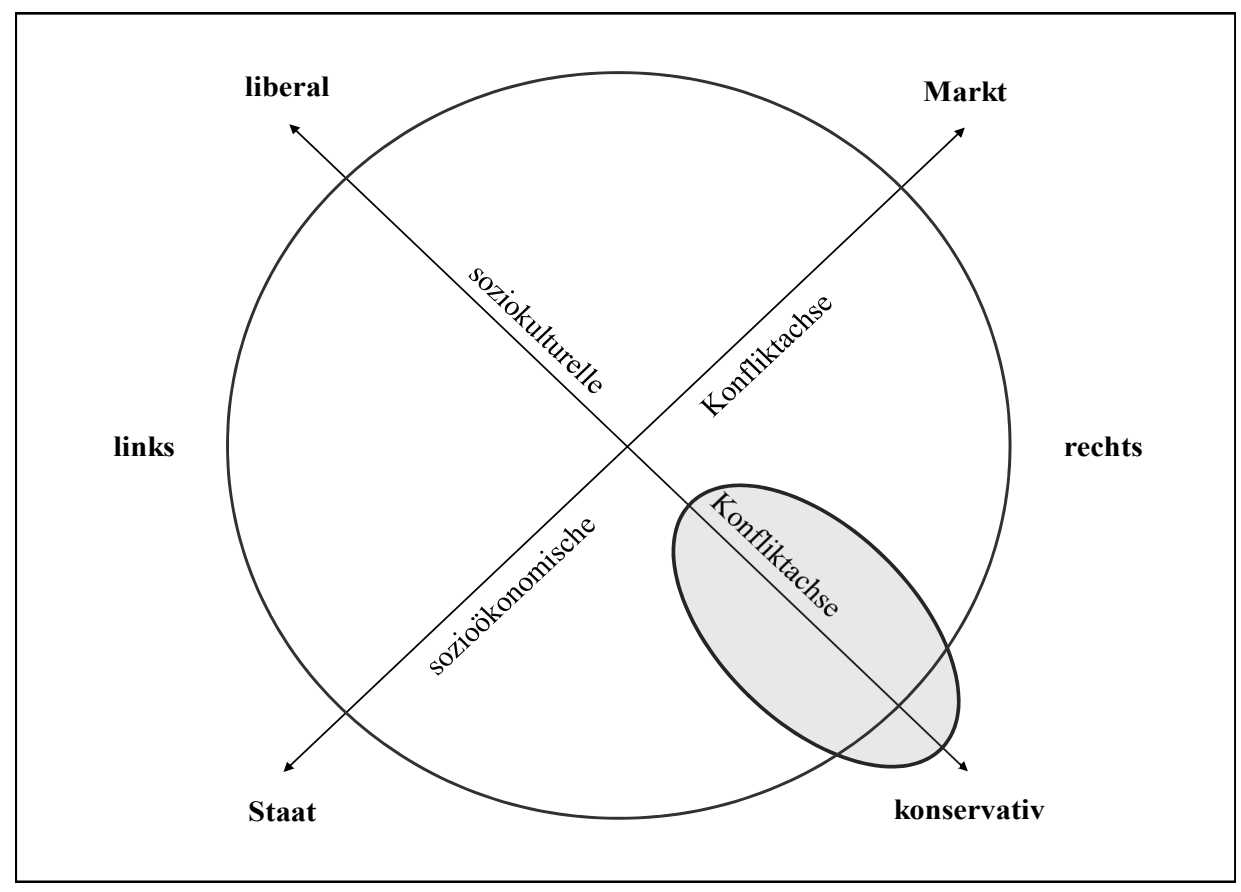

Quelle: Eigene Darstellung

15 Vgl. Frank Decker, Parteiendemokratie im Wandel, 2. Aufl. 2018, S. 55. 
auf beiden Achsen nicht übereinstimmen muss, macht gerade den Nutzen des zweiteiligen Schemas aus. So ist es beispielsweise ein Charakteristikum vieler liberaler Parteien, dass sie auf der kulturellen Achse eher links und auf der ökonomischen Achse eher rechts anzutreffen sind. Umgekehrt gilt dasselbe für die am äußeren rechten Pol der kulturellen Konfliktachse platzierten Rechtspopulisten. Waren deren sozial- und wirtschaftspolitische Positionen in der Entstehungsphase noch überwiegend marktliberal geprägt, sind sie heute in einigen Ländern die vehementesten Befürworter des Wohlfahrtsstaates.

Das Missverständnis, wonach die Wertepolitik aus dem Links-Rechts-Schema herausfällt, geht auf den US-amerikanischen Sozialwissenschaftler Ronald Inglehart zurück. Dessen Anfang der siebziger Jahre entwickelte Theorie des "postmaterialistischen « Wertewandels operationalisierte die materiellen und immateriellen Werte so, dass die ersteren ausschließlich mit rechten und die letzteren mit linken Positionen zusammenfielen. ${ }^{16}$ Inglehart selbst machte dabei aus seiner Sympathie für den linken Postmaterialismus keinen Hehl. Dass seine Zuordnung wenig sinnvoll war, sollte sich spätestens mit dem Aufkommen der neuen Rechtsparteien in den achtziger Jahren erweisen. Denn deren Agenda war genauso von wertebezogenen Themen geprägt wie jene der Neuen Linken in den siebziger Jahren, auf die sie teilweise eine unmittelbare Reaktion darstellte. Die Neue Rechte gehörte damit ebenfalls zum "postmaterialistischen « Teil des politischen Spektrums, nur dass sie eben ganz andere - konservative oder autoritäre - Positionen vertrat.

Stimmt diese Interpretation, dann basiert die Entstehung der grünen Parteien ebenso wenig auf eine »neuen « Konfliktlinie wie das Aufkommen des Rechtspopulismus. Beide Erscheinungen stellen vielmehr die Folge eines veränderten politischen Themenhaushalts dar, der die Prioritäten sowohl innerhalb der verteilungs- und wertebezogenen Sphäre als auch zwischen diesen verschoben hat. ${ }^{17}$ Gleichzeitig haben sich die Kontexte der Konflikte räumlich und zeitlich ausgeweitet. Blieb die verteilungspolitische Auseinandersetzung bis in die sechziger Jahre hinein noch weitgehend auf die soziale Frage im nationalstaatlichen Rahmen beschränkt (in Abhängigkeit von der Position im Erwerbsprozess), erstreckte sie sich nun auch auf die ökonomische Benachteiligung der Dritten Welt und die Benachteiligung künftiger Generationen im Zuge der ökologischen Krise. Dies stellte vor allem die sozialdemokratische Mainstream-Linke vor ein Problem, die versuchen musste, die aus den verschiedenen Gleichheitskontexten resultierenden Interessenkonflikte - zwischen nationalen und internationalen Ver-

16 Vgl. Ronald Inglehart, The Silent Revolution. Changing Values and Political Styles Among Western Publics, Princeton 1977. Als Indikatoren für Materialismus wird der Kampf gegen steigende Preise und die Aufrechterhaltung der Ordnung herangezogen, als Indikatoren für Postmaterialismus der Schutz der freien Meinungsäußerung und das Mitspracherecht bei wichtigen Entscheidungen.

17 Der Umweltschutz ist dabei trotz seiner Wertebasiertheit primär der sozioökonomischen Konfliktachse zuzuordnen, da er - je nach Bereich unterschiedlich weitreichende - staatliche Eingriffe in das Marktgeschehen erfordert. 
pflichtungen, in- und ausländischen Arbeitnehmern sowie der gegenwärtigen und künftigen Generation - auszubalancieren.

Die vergangenen und aktuellen innenpolitischen Debatten in der Bundesrepublik um die Vertiefung der europäischen Integration, die Zukunftssicherung des Sozialstaats, die Erreichung der Klimaziele und nicht zuletzt die Migrations- und Flüchtlingspolitik - machen deutlich, dass diese Konflikte weiter zugenommen haben. Wie unübersichtlich die Frontverläufe dabei geworden sind, zeigen etwa die heftigen Auseinandersetzungen in der Partei Die Linke, wo eine Minderheit unter Führung von Sahra Wagenknecht und Oskar Lafontaine die flüchtlingsfreundliche Linie der Parteispitze offen in Frage stellt. Ihre Forderung nach einer Begrenzung der Zuwanderung erscheint auf den ersten Blick wie eine Anleihe beim Rechtspopulismus. Der Schutz des einheimischen Arbeitsmarkts und Sozialstaats vor migrationsbedingten Überforderungen lässt sich aber von einem linken Standpunkt aus gut begründen - zumal wenn er mit der Anerkennung internationaler Verpflichtungen (bei der Fluchtursachenbekämpfung und heimatnahen Unterbringung der Flüchtlinge) einhergeht. ${ }^{18}$

Trifft diese Feststellung zu, dann würde vieles dafür sprechen, den Populismus nicht nur oder primär als Folge eines kulturellen Konflikts zu deuten, sondern auch als ökonomisches Phänomen. ${ }^{19}$ Der Blick auf die europäische Landkarte legt eine solche Interpretation nahe. Er zeigt, dass in den west- und nordeuropäischen Ländern hauptsächlich die rechtspopulistischen Parteien reüssieren, während in Südeuropa der Linkspopulismus dominiert. Bei der erstgenannten Gruppe handelt es sich um wettbewerbsstarke, offene Volkswirtschaften, die ein hohes Wohlfahrtsstaatsniveau und zugleich einen hohen Migrantenanteil in der Bevölkerung aufweisen. Wertebezogene Konflikte über die kulturelle Zugehörigkeit der Zuwanderer verquicken sich hier mit verteilungsbezogenen Auseinandersetzungen um Löhne und staatliche Leistungen, die vor allem das untere Drittel oder Viertel der Bevölkerung, aber auch die um ihren Abstieg fürchtenden Mittelschichten betreffen. Dies gilt zumal, als der Wohlfahrtsstaat durch den globalen Wettbewerb - dessen Verlierer er eigentlich schützen soll - selbst unter Druck gerät.

Wie das ökonomische und kulturelle Cleavage genau zusammenwirken, bleibt unter den Interpreten des Rechtspopulismus freilich umstritten. Philip Manows Feststellung, die kulturellen Konflikte dienten lediglich dazu, die ökonomischen Probleme »aufzuladen ${ }^{20}$, um sie dadurch politisch zu mobilisieren, dürfte hier in zweierlei Hinsicht zu kurz greifen. Zum einen setzt sich das kulturelle Cleavage aus unterschiedlichen Streitfragen und Unterkonflikten zusammen, von denen verteilungspolitisch nicht alle die gleiche Relevanz besitzen wie die Zuwanderung. Die rechtliche Gleichstellung von Homosexuellen nimmt zum Beispiel niemanden etwas weg. Zum anderen behalten die wertebezogenen Konflikte auch jenseits der verteilungspolitischen Aspekte eine eigen-

18 Vgl. Paul Collier, »Asylpolitik neu denken. Vorschläge für ein gescheitertes System « in: Ursula Bitzegeio u.a. (Hg.), Flucht-Transit-Asyl, Bonn 2018, S. 35-43.

19 Vgl. Philip Manow, Die Politische Ökonomie des Populismus, Berlin 2018.

20 Manow, Politische Ökonomie, aaO. (FN 19), S. 16. 
ständige Bedeutung - und zwar umso mehr, je größer die kulturellen Unterschiede zwischen den Zuwanderern und der sie aufnehmenden Mehrheitsgesellschaft sind. ${ }^{21}$

Hauptverlierer des Parteiensystemwandels sind die Volksparteien. Weil sie auf beiden Konfliktachsen »mittige und zum Teil voneinander wenig unterscheidbare Positionen vertreten, leiden sie unter der Polarisierung, während die ideologisch radikaleren Vertreter, die näher an den Enden der jeweiligen Pole angesiedelt sind - also auf der ökonomischen Achse besonders FDP und Linke, auf der kulturellen Achse Grüne und AfD - profitieren.

Unterschiedliche Meinungen gibt es, wie mit diesem Dilemma am besten umzugehen sei. In der Union wird dieser Streit vor allem über der Flüchtlingspolitik ausgetragen, wo die einen dafür plädieren, die »offene Flanke« gegenüber der AfD zu schließen, während die anderen fürchten, durch eine Preisgabe liberaler Positionen Wähler in der Mitte an SPD und die Grünen zu verlieren. Bei den Sozialdemokraten stehen wiederum auf der einen Seite diejenigen, die die linksliberalen Positionen der Partei auf der kulturellen Achse zurückdrängen und sie gleichzeitig sozial- und wirtschaftspolitisch wieder stärker nach links rücken möchten.22 Die anderen raten dagegen, den Spagat auszuhalten und sich um eine programmatische und strategische Zusammenführung der auseinanderstrebenden Wählermilieus zu bemühen.

Wie immer sich die beiden Noch-Volksparteien neu positionieren, dürfte es ihnen schwerfallen, in einem sich verfestigenden Sechsparteiensystem nennenswerte Wähleranteile von der Konkurrenz zurückzuerobern. Verschärft wird das Problem durch die erzwungene Neuauflage der Großen Koalition. Diese treibt unzufriedene Wähler automatisch in die Arme der Opposition - auch der rechtspopulistischen. Unter diesen Bedingungen wäre es bereits ein Erfolg, wenn es Union und SPD in den kommenden Jahren gelänge, durch ein halbwegs vernünftiges Regieren die AfD zumindest einzudämmen und eine weitere Zuspitzung der kulturellen und ökonomischen Konflikte zu verhindern.

$21 \mathrm{Vgl} \mathrm{Ruud} \mathrm{Koopmans,} \mathrm{Assimilation} \mathrm{oder} \mathrm{Multikulturalismus?} \mathrm{Bedingungen} \mathrm{gelungener} \mathrm{Inte-}$ gration, Berlin 2017.

22 Vgl. z.B. Nils Heisterhagen, Die liberale Illusion. Warum wir einen linken Realismus brauchen, Bonn 2018. 